\title{
Optimal Queue Scheduling for Hybrid Cognitive Radio Maintaining Maximum Average Service Rate Under Delay Constraints
}

\author{
Jie Hu, Lie-Liang Yang, Lajos Hanzo \\ School of Electronics and Computer Science, University of Southampton, Southampton, UK, SO17 1BJ, \\ Tel: +44-23-8059 3125, Fax: +44-23-8059 4508 \\ Email: \{jh10g11, lly, lh\}@ecs.soton.ac.uk, http://www-mobile.ecs.soton.ac.uk
}

\begin{abstract}
As a promising technique of improving the attainable bandwidth efficiency, cognitive radio (CR) has attracted substantial attention from both the academic and industrial communities. In order to improve the performance of the secondary user (SU), a novel hybrid CR system is introduced, which combines the conventional interweave and underlay paradigms for enhancing the chances of the SU to access the spectrum. Queuing theory is invoked in this paper for analysing the impact of the primary user's (PU) delay tolerance on the performance of the SU. Multiple queues are assumed for the SU engaging in video communication. Besides the Poisson traffic generation, we model a Rayleigh fading channel as a Poisson service process with the aid of the outage probability in the presence of cochannel interferences. Two valuable goals are achieved, namely that of maximizing the average service rate and minimizing the overall average delay of the SU's multiple queues. As our numerical results demonstrate, the overall average delay of the $\mathrm{SU}$ becomes $27 \%$ and $34 \%$ lower than that of the Proportional as well as that of the Round-Robin schemes respectively.
\end{abstract}

\section{INTRODUCTION}

Cognitive Radio (CR) [1] constitutes an attractive technique of improving the bandwidth efficiency in wireless communications. Two kinds of customers are supported in this system, namely the primary user (PU) and the secondary user (SU). Furthermore, three paradigms are used in the existing CR systems, namely the overlay, the underlay and the interweave paradigms [2]. In overlay systems, the SUs invoke sophisticated signal processing and coding to maintain or improve the communication of primary users (PUs), while also obtaining some additional bandwidth for their own communication. Here we focus our attention on the other two paradigms. According to the interweave paradigm, the PUs are authorized to access the radio spectrum at will, while the SUs are only able to access it when the PUs release it. In the scenario that the PUs request resources, even if a SU's session is already under way, it must be paused temporarily until the PUs complete their transmissions. According to the underlay paradigm, the PUs and SUs are allowed to transmit their data at the same time. An interference threshold is set up at the PUs in order

The financial support of the RC-UK's India-UK Advanced Technology Centre (IU-ATC), that of the EU's concerto project and of the China Scholarship Council (CSC) is gratefully acknowledged to guarantee that the PU's link is not unduly interfered by the SUs. Their transmission would only be terminated, if the interference imposed by the SU exceeds a threshold.

Some previous work exists in this area. Du and Zhang [3] proposed an adaptive resource allocation scheme for multilayer video streaming in wireless unicast/multicast services. In the scenario of a single queue conceived for the SU, the maximum throughput of the SU has been investigated in [4] under the interweave paradigm by finding the optimal transmit power of the SU. The authors of [5] then extended this research to a CR system supporting multiple PUs and a single $\mathrm{SU}$ in order to find the optimal transmit power and to characterize the relaying probability of the SU. The stability region of a CR network supporting multiple PUs, multiple relays and multiple SUs has been analysed in [6]. The authors of [4] to [6] employed the interweave paradigm, where the SUs have fewer opportunities to access the resources. All these studies assumed that only a single queue was set up for each of the users, which fails to realistically represent the characteristics of multimedia video communication, and the results were conceived under the assumption of queuing stability, where the specific QoS constraints such as the delay tolerance were completely ignored.

Against this background, our novel contributions are as follows.

1) A hybrid CR is proposed by amalgamating the interweave and underlay paradigms for enhancing the opportunities that the SU can access the system.

2) The optimal weighting coefficient $\varepsilon$ of the hybrid interweave/underlay scheme maximizing the average service rate of the $\mathrm{SU}$ is determined.

3) By exploiting the Lagrange method, we are able to minimize the overall average delay for multiple video streams of the SU.

This paper is organized as follows. In Section II, our system model including the MAC and physical layer is described, while in Section III the related queuing analysis is carried out for both the PU and SU; In Section IV, the problem of finding the optimal solution is formulated and solved, which is then followed by our numerical results in Section V. Finally, our 


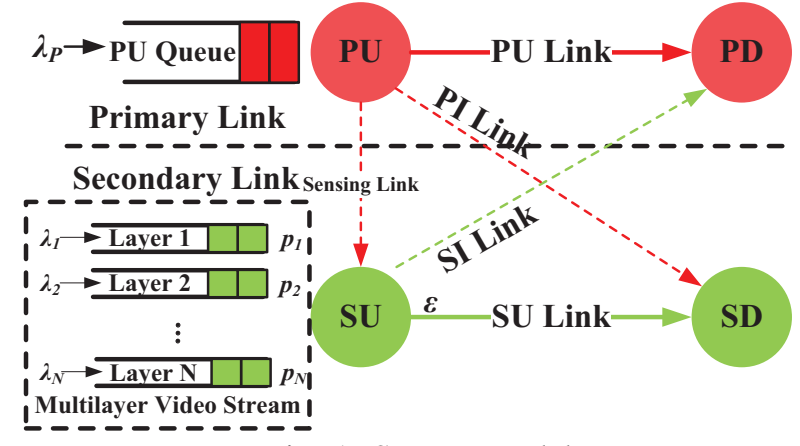

Fig. 1: System model

conclusions are offered in Section VI.

\section{System Model}

Fig.1 portrays a CR system supporting a PU source and PU destination (PD) pair as well as a SU source and SU destination (SD) pair. A novel hybrid CR policy is employed. Given a spectral band, the SU first senses the activity of the PU. If this sensing is reliable, the SU may access the band with a unity probability, whenever the PU is idle. Furthermore the SU may access the band with a probability of $\varepsilon$ when PU is busy under the constraint that QoS of the PU must be guaranteed. Given the fact that the SU tends to roam in the vicinity of the PU, which indicates a reliable link between the $\mathrm{PU}$ and SU, perfect sensing is assumed for simplicity. Let us now consider the details of the MAC and physical layers.

\section{A. MAC Layer Model}

Observe from Fig. 1 that a buffer is provided for the PU for storing the packets which cannot be transmitted immediately. In order to support video communications for the SU, multiple buffers are provided for accommodating the multiple queues generated by multi-layer video encoding [7].

The basic time unit in our system is a time slot (TS). The packets' arrival at the PU's buffer follows the Poisson process with a mean of $\lambda_{P}$ packets/TS. There exits a QoS constraint for the PU, which is the response time (including both the transmission and buffering time) that should not exceed $T_{P}$, namely the maximum tolerable delay of the PU. The packets' arrival at the buffers of the SU also follow the classic Poisson process with means of $\left\{\lambda_{i}, i=1,2, \ldots N\right\}$ packets/TS. Regardless, whether the PU is idle or busy, if the SU has already successfully accessed the system, we should determine the probability of each queue of the SU completing its transmission of the stored packets. These probabilities may be denoted as $\left\{p_{i}, i=1,2, \ldots, N\right\}$. No specific QoS constraints are imposed on the SU. However, one of our primary goal is to find an optimal scheduling scheme $\left\{p_{i}\right\}$ and hybrid parameter $\varepsilon$, which jointly minimize the overall average delay of all the SU's queues.

When the signal to interference plus noise ratio (SINR) is above a specific threshold, the packet at the head of the buffer can be successfully transmitted. From the perspective of queuing, we refer to this as the service process. In line with classic probability theory [8], if the service process of a packet follows the Bernoulli distribution, the scenario associated with multiple packets may be approximately modelled as a Poisson process whose average service rate equals to the successful transmission probability for a single packet. This approximation enables us to utilize the outage probability experienced in a wireless channel for modelling the packets' service process.

\section{B. Physical Layer Model}

Corresponding to the related research in [9], the multipacket reception (MPR) model conceived for the physical layer is introduced to analyse the queuing performances achieved in a collision-contaminated channel, including the following five conditional probabilities:

$q_{P U|P U|}^{(P D)}:$ only the PU's packet is received successfully at the PD when only the PU transmits.

$q_{P U \mid\{P U, S U\}}^{(P D)}$ : only the PU's packet is received successfully at the PD when both the PU and SU transmit.

$q_{S U \mid S U\}}^{(S D)}$ : only the SU's packet is received successfully at the SD when only the SU transmits.

$q_{S U \mid\{S U, P U\}}^{(S D)}$ : only the SU's packet is received successfully at $\mathrm{SD}$ when both SU and PU transmit.

$q_{P U \mid\{U\}}^{(S U)}:$ only the PU's packet is received successfully at the SU when only the PU transmits. Since we assume perfect spectrum sensing, this probability is always equal to 1 .

The radio propagation between any pair of nodes is assumed to be affected by independent stationary Rayleigh flat-fading channels $h_{i}(t)$ associated with $E\left[\left|h_{i}(t)\right|^{2}\right]=1$ ( $t$ denotes time slot).The cumulative distribution function of the instantaneous power $\left|h_{i}(t)\right|^{2}$ is then given by [8]:

$$
P\left[\left|h_{i}(t)\right|^{2}<x\right]=1-\exp (-x) .
$$

The average channel power gain (due to shadowing and path loss) and the SINR threshold are denoted by $\gamma_{i}$ and $\beta_{i}$ respectively, where $i$ represents "P" for the PU link, as shown in Fig.1, "S" for the SU link, "PI" for the PI link imposing interference from the PU on the SD, "SI" for the SI link inflicting interference by the SU upon the PD, and "PS" for the sensing link spanning from PU to SU. Without loss of generality, the transmit power of both the PU and SU is normalized by the noise power, which are denoted by $P_{P}$ and $P_{S}$, respectively.

By deriving the outage probability of the noisecontaminated Rayleigh channel in the absence of any interference, we may get $q_{P U \mid\{P U\}}^{(P D)}$ and $q_{S U \mid\{S U\}}^{(S D)}$ defined at the beginning of this section:

$$
\begin{aligned}
& q_{P U \mid\{P U\}}^{(P D)}=1-P\left[\gamma_{P}\left|h_{P}(t)\right|^{2} P_{P}<\beta_{P}\right]=\exp \left(-\frac{\beta_{P}}{\gamma_{P} P_{P}}\right), \\
& q_{S U \mid\{S U\}}^{(S D)}=1-P\left[\gamma_{S}\left|h_{S}(t)\right|^{2} P_{S}<\beta_{S}\right]=\exp \left(-\frac{\beta_{S}}{\gamma_{S} P_{S}}\right) .
\end{aligned}
$$

It is however less straightfoward to derive the other two conditional probabilities, namely $q_{P U \mid\{P U, S U\}}^{(P D)}$ and $q_{S U \mid\{S U, P U\}}^{(S D)}$ owing to the effects of cochannel interferences imposed on the PD and SD. Let us assume that $X_{0}, \ldots, X_{n}$ are independent 
exponentially distributed random variables associated with the respective means of $E\left[X_{j}\right]=1 / \sigma_{j}, j=0, \ldots n$, and $A>0, B>0$ are constants. Then according to [10], the problem at hand is equivalent to solving the following probability evaluation:

$$
P\left(X_{0}>A+B \sum_{j=1}^{n} X_{j}\right)=\exp \left(-\sigma_{0} A\right) \prod_{j=1}^{n} \frac{1}{1+\frac{\sigma_{0} B}{\sigma_{j}}} .
$$

Next we will derive the expressions of $q_{P U \mid\{P U, S U\}}^{(P D)}$ and $q_{S U \mid\{S U, P U\}}^{(S D)}$ in the realistic scenario, where both the effects of Gaussian noise and of the cochannel interference are considered. Given

$$
\begin{aligned}
q_{P U \mid\{P U, S U\}}^{(P D)} & =1-P\left[\gamma_{P}\left|h_{P}(t)\right|^{2} P_{P}<\beta_{P}\left(1+\gamma_{S I}\left|h_{S I}(t)\right|^{2} P_{S}\right)\right] \\
& =P\left[\left|h_{P}(t)\right|^{2}>\frac{\beta_{P}}{\gamma_{P} P_{P}}+\frac{\beta_{P} \gamma_{S I} P_{S}}{\gamma_{P} P_{P}}\left|h_{S I}(t)\right|^{2}\right] \\
q_{S U \mid\{S U, P U\}}^{(S D)} & =1-P\left[\gamma_{S}\left|h_{S}(t)\right|^{2} P_{S}<\beta_{S}\left(1+\gamma_{P I}\left|h_{P I}(t)\right|^{2} P_{P}\right)\right] \\
& =P\left[\left|h_{S}(t)\right|^{2}>\frac{\beta_{S}}{\gamma_{S} P_{S}}+\frac{\beta_{S} \gamma_{P I} P_{P}}{\gamma_{S} P_{S}}\left|h_{P I}(t)\right|^{2}\right]
\end{aligned}
$$

and assuming $n=1$ in Equation (4), we may finally obtain the closed-form expressions of $q_{P U \mid\{P U, S U\}}^{(P D)}$ and $q_{S U \mid\{S U, P U\}}^{(S D)}$ from the above two equations, which are

$$
\begin{aligned}
& q_{P U \mid\{P U, S U\}}^{(P D)}=\exp \left(-\frac{\beta_{P}}{\gamma_{P} P_{P}}\right) \frac{1}{1+\beta_{P} \gamma_{S I} P_{S} /\left(\gamma_{P} P_{P}\right)}, \\
& q_{S U \mid\{S U, P U\}}^{(S D)}=\exp \left(-\frac{\beta_{S}}{\gamma_{S} P_{S}}\right) \frac{1}{1+\beta_{S} \gamma_{P I} P_{P} /\left(\gamma_{S} P_{S}\right)} .
\end{aligned}
$$

Since perfect spectrum sensing is assumed, the sensing link between the PU and the SU is not contaminated by any noise or interference, which implies that the conditional probability of $q_{P U \mid\{P U\}}^{(S U)}$ defined above is always equal to one.

\section{Queuing Analysis}

In this section, the following two goals will be achieved:

1) A new dominant system is defined for characterizing the inter-dependence of the PU's and SU's queue;

2) Throughout our queueing analysis related to both the PU and SU, we derive the expression of the QoS constraint for the PU and the average service rates of both the PU and SU.

Let us commence from the following two features of the $\mathrm{M} / \mathrm{M} / 1$ queuing system. The queue is empty with a probability of $P[Q=0]=1-\lambda / \mu$ and the average response time is $T=1 /(\mu-\lambda)$.

According to our discussions in Section II, all the packets' arrival processes of the hybrid CR system are Poisson distributed. Additionally, given the successful packet's transmission probabilities, the packets' departure processes of both the primary and secondary link may be approximately modelled by the Poisson distribution. These make the $\mathrm{M} / \mathrm{M} / 1$ queuing analysis readily applicable to our system.

\section{A. Dominant System}

The average service rates of the PU and SU depend on each other's queue sizes. Hence these queues are inter-dependent.
Consequently the rates of the individual service processes cannot be computed directly. For the sake of circumventing this problem, the stochastic dominance principle of [11] is utilized.

The dominant system was defined by allowing a set of terminals having no packets in their transmit-buffer to continue transmitting dummy packets. In this manner, the queues in the dominant system stochastically dominate the queues of the original system. Given the same initial conditions, the sizes of the queues in the dominant system are not smaller than that of the original system. This dominant system is defined as:

1) If $Q_{S}=0$, the $\mathrm{SU}$ transmits dummy packets with a unity probability, when $Q_{P}=0$;

2) If $Q_{S}=0$, the SU transmits dummy packets with a probability of $\varepsilon$, when $Q_{P} \neq 0$.

Here $Q_{S}=0$ represents that all the SU's queues are empty, and $Q_{P}$ denotes the size of the PU's queue.

\section{B. Queuing Analysis of the PU and $S U$}

Under the assumption of encountering the above mentioned dominant system, we can derive the probability of successfully transmitting one of the PU's packets, which is also the average service rate of the Poisson service process given by:

$$
\mu_{P}=q_{P U \mid\{P U\}}^{(P D)} \cdot(1-\varepsilon)+q_{P U \mid\{P U, S U\}}^{(P D)} \cdot \varepsilon .
$$

Let us assume that the average service rate is $\mu_{P}$, the average arrival rate is $\lambda_{P}$ and that our QoS constraint is formulated as having a response time below $T_{P}$. We will also refer to $T_{P}$ as delay tolerance. According to classic $\mathrm{M} / \mathrm{M} / 1$ queuing analysis [8], the following condition must be obeyed:

$$
0 \leq \lambda_{P} \leq \mu_{P}-\frac{1}{T_{P}} .
$$

We may denote the probabilities of the PU's queue being empty or not by $\lambda_{P}$ and $\mu_{P}$. Consequently we may derive the probability of successfully transmitting one of the SU's packets, which is also equivalent to the average service rate $\mu_{S}$ of the Poisson service process as follows:

$$
\begin{aligned}
\mu_{S} & =q_{S U \mid\{S U\}}^{(S D)} \cdot P\left[Q_{P}=0\right]+q_{S U \mid\{S U, P U\}}^{(S D)} \cdot P\left[Q_{P} \neq 0\right] \varepsilon \\
& =q_{S U \mid\{S U\}}^{(S D)}-\lambda_{P} \cdot \frac{q_{S U \mid\{S U\}}^{(S D)}-q_{S U \mid\{S U, P U\}}^{(S D)} \cdot \varepsilon}{q_{P U \mid\{P U\}}^{(P D)}-\varepsilon \cdot\left[q_{P U \mid\{P U\}}^{(P D)}-q_{P U \mid\{P U, S U\}}^{(P D)}\right]} .
\end{aligned}
$$

\section{Problem Formulation}

Here, based on our queuing analysis conducted in Section III, two associated problems will be solved.

1) Finding the optimal hybrid parameter $\varepsilon^{*}$ to maximize the average service rate $\mu_{S, \max }$ of the SU;

2) Given $\mu_{S, \max }$, the most suitable queue scheduling scheme is proposed for minimizing the overall average delay of all the SU's queues.

\section{A. Maximum Average Service Rate of the $S U$}

The optimal hybrid parameter $\varepsilon^{*}$, which determines the weighting factor of the interweave and underlay priorities in 
the CR system, must be found for maximizing the average service rate of the SU without violating the QoS constraint of the PU. We may find this solution by constructing the problem P1 as follows:

\section{Problem 1: P1}

$$
\begin{aligned}
& \arg \max _{\varepsilon} \mu_{S}(\varepsilon), \\
& 0 \leq \varepsilon \leq \frac{q_{P U \mid\{P U\}}^{(P D)}-\lambda_{P}-\frac{1}{T_{P}}}{q_{P U \mid\{P U\}}^{(P D)}-q_{P U \mid\{P U, S U\}}^{(P D)}} .
\end{aligned}
$$

In order to solve $\mathbf{P 1}$, we should differentiate $\mu_{S}(\varepsilon)$ with respect to $\varepsilon$, yielding:

$$
\begin{gathered}
\frac{d \mu_{S}}{d \varepsilon}=\frac{\lambda_{P} \cdot \alpha}{\left[q_{P U \mid\{P U\}}^{(P D)}-\varepsilon\left(q_{P U \mid\{P U\}}^{(P D)}-q_{P U \mid\{P U, S U\}}^{(P D)}\right)\right]^{2}} \\
\alpha=q_{S U \mid\{S U, P U\}}^{(S D)} q_{P U \mid\{P U\}}^{(P D)}+q_{S U \mid\{S U\}}^{(S D)} q_{P U \mid\{P U, S U\}}^{(P D)}-q_{S U \mid\{S U\}}^{(S D)} q_{P U \mid\{P U\}}^{(P D)}
\end{gathered}
$$

According to the different values of $\alpha$ and $\lambda_{P}$, we have several different scenarios deserving further discussions in order to derive the maximum average service rate $\mu_{S, \max }\left(\varepsilon^{*}\right)$ of the SU achieved with the aid of the optimal $\varepsilon^{*}$ :

\section{Case 1:}

If we have $\alpha>0$, which implies that $\frac{d \mu_{S}}{d \varepsilon}>0$, then $\mu_{S}(\varepsilon)$ is a monotonically increasing function of $\varepsilon$. From the constraint imposed by (8), we infer that provided we have $0 \leq \varepsilon \leq 1$, when $\varepsilon=1$, the right side of (8) is at its minimum value. This constraint may be rewritten as:

$$
0 \leq \lambda_{P} \leq q_{P U \mid\{P U, S U\}}^{(P D)}-\frac{1}{T_{P}} .
$$

If the constraint (13) holds, we may conclude that $\varepsilon^{*}=1$, which represents the full underlay paradigm in our hybrid CR system, hence maximizing the average service rate of the SU. Consequently the maximum average service rate is

$$
\mu_{S, \max }=q_{S U \mid\{S U\}}^{(S D)}-\frac{\lambda_{P}}{q_{P U \mid\{P U, S U\}}^{(P D)}} \cdot\left(q_{S U \mid\{S U\}}^{(S D)}-q_{S U \mid\{S U, P U\}}^{(S D)}\right) .
$$

\section{Case 2:}

If both $\alpha>0$ and $\lambda_{P}>q_{P U \mid\{P U, S U\}}^{(P D)}-\frac{1}{T_{P}}$ holds, then upon taking the constraint (10) into consideration, $\mu_{S}(\varepsilon)$ is seen to be a monotonically increasing function of $\varepsilon$. Hence we can derive the optimal value of $\varepsilon^{*}$, yielding:

$$
\varepsilon^{*}=\frac{q_{P U \mid\{P U\}}^{(P D)}-\lambda_{P}-\frac{1}{T_{P}}}{q_{P U \mid\{P U\}}^{(P D)}-q_{P U \mid\{P U, S U\}}^{(P D)}},
$$

which represents the partial interweave and underlay paradigms in our hybrid CR system. Therefore the maximum average service rate of the $\mathrm{SU}$ in this case becomes

$$
\begin{aligned}
\mu_{S, \max } & =q_{S U \mid\{S U\}}^{(S D)}-\frac{\lambda_{P}}{\lambda_{P}+1 / T_{P}} \\
& \cdot\left(q_{S U \mid\{S U\}}^{(S D)}-\frac{q_{S U \mid\{S U, P U\}}^{(S D)}\left(q_{P U \mid\{P U\}}^{(P D)}-\lambda_{P}-1 / T_{P}\right)}{q_{P U \mid\{P U\}}^{(P D)}-q_{P U \mid\{P U, S U\}}^{(P D)}}\right) .
\end{aligned}
$$

\section{Case 3:}

If we have $\alpha \leq 0$, which indicates that $\frac{d \mu_{S}}{d \varepsilon} \leq 0, \mu_{S}(\varepsilon)$ becomes a monotonically decreasing function of $\varepsilon$. Then the optimal value is $\varepsilon^{*}=0$, which corresponds to the full interweave paradigm in our hybrid cognitive radio system, hence maximizing the average service rate of the SU:

$$
\mu_{S, \max }=q_{S U \mid\{S U\}}^{(S D)}-\lambda_{P} \cdot \frac{q_{S U \mid\{S U\}}^{(S D)}}{q_{P U \mid\{P U\}}^{(P D)}} .
$$

\section{B. Optimal Scheduling among Multilayer Queues at Sec-} ondary User

Let us now detail further the derivation of the optimal queue scheduling scheme $\left\{p_{i}^{*}, i=1,2, \ldots, N\right\}$ conceived for minimizing the overall average delay of the SU's multiple queues. Given the associated maximum average service rate $\mu_{S, \max }$ of the SU, we construct the problem $\mathbf{P 2}$ as:

Problem 2: P2

$$
\arg \min _{\left\{p_{1}, p_{2}, \ldots, p_{N}\right\}}\left\{\frac{1}{N} \sum_{i=1}^{N} \frac{1}{p_{i} \cdot \mu_{S, \max }-\lambda_{i}}\right\},
$$

Subject to:

$$
\begin{aligned}
& \sum_{i=1}^{N} p_{i}=1, \\
& \lambda_{i}-p_{i} \cdot \mu_{S, \max }<0 .
\end{aligned}
$$

It may then be readily seen that:

1) the objective function (18) is convex over $\left\{p_{i}, i=\right.$ $1,2, \ldots, N\}$;

2) the functions on the left-hand side of both the equality and inequality constraints shown in (19) and (20) are affine over $\left\{p_{i}, i=1,2, \ldots, N\right\}$.

Hence, $\mathbf{P 2}$ is a convex problem [13] and the optimal solution can be obtained with the aid of the Lagrangian method and the Karush-Kuhn-Tucker (KKT) conditions [13], where the KKT conditions were proposed in [13] to solve convex optimization problems similar to ours under an inequality constraint.

The Lagrangian function is constructed as:

$J=\frac{1}{N} \sum_{i=1}^{N} \frac{1}{p_{i} \mu_{S, \max }-\lambda_{i}}+\sum_{i=1}^{N} \varphi_{i}\left(\lambda_{i}-p_{i} \mu_{S, \max }\right)+\eta\left(\sum_{i=1}^{N} p_{i}-1\right)$,

where $\eta \geq 0$ and $\varphi_{i} \geq 0, i=1,2, \ldots, N$, are the Lagrangian multipliers associated with conditions (19) and (20), respectively. Then, the optimal $\left\{p_{i}^{*}, i=1,2, \ldots, N\right\}$ and the Lagrangian multipliers of the optimization problem $\mathbf{P 2}$ satisfy the following KKT conditions [13]:

$$
\begin{cases}\left.\frac{\partial J}{\partial p_{i}}\right|_{p_{i}=p_{i} *}=0, & i=1,2, \ldots N \\ \varphi_{i}^{*} \geq 0 \text { and } \eta^{*} \geq 0, & i=1,2, \ldots N ; \\ \left.\frac{d J}{d \eta}\right|_{p_{i}=p_{i} *}=\sum_{i=1}^{N} p_{i}-1=0, & i=1,2, \ldots N \\ \varphi_{i}^{*}\left(\lambda_{i}-p_{i}^{*} \mu_{S, \max }\right)=0, & i=1,2, \ldots N .\end{cases}
$$


Taking the derivative of $J$ with respect to $p_{i}$, we arrive at

$$
\frac{\partial J}{\partial p_{i}}=\frac{1}{N} \frac{-\mu_{S, \max }}{\left(p_{i} \mu_{S, \max }-\lambda_{i}\right)^{2}}-\varphi_{i} \mu_{S, \max }+\eta .
$$

Substituting Equation (23) into the first line of Equation (22) and solving for $p_{i}^{*}$ under the constraint (20), we have

$$
p_{i}^{*}=\frac{1}{\sqrt{N \mu_{S, \text { max }}\left(\eta-\varphi_{i}^{*} \mu_{S, \max }\right)}}+\frac{\lambda_{i}}{\mu_{S, \text { max }}} .
$$

Naturally, $\lambda_{i}-p_{i}^{*} \mu_{S, \max }<0$ must hold according to the constraint defined in Equation (20). Therefore, the only solution satisfying the fourth line of Equation (22) is $\varphi_{i}^{*}=0$. Substituting $\varphi^{*}=0$ into Equation (24), we may rewrite $p_{i}^{*}$ of (24) as

$$
p_{i}^{*}=\frac{1}{\sqrt{N \eta^{*} \mu_{S, \max }}}+\frac{\lambda_{i}}{\mu_{S, \max }} .
$$

All we have to do now is to find the value of $\eta^{*}$. Substituting Equation (25) into the third line of Equation (22), we arrive at:

$$
\eta^{*}=\frac{N \cdot \mu_{S, \max }}{\left(\mu_{S, \max }-\sum_{i=1}^{N} \lambda_{i}\right)^{2}}
$$

Substituting $\eta^{*}$ of Equation (26) into Equation (25), we finally arrive at the optimal queue scheduling scheme characterized by

$$
p_{i}^{*}=\frac{\mu_{S, \max }-\sum_{i=1}^{N} \lambda_{i}+N \lambda_{i}}{N \cdot \mu_{S, \max }} .
$$

Some special cases of interest are as follows:

1) The SU has a single data stream associated with $N=1$, and the average arrival rate of it is $\lambda_{S}$. Substituting these into Equation (27), the queue scheduling scheme is characterized by $p^{*}=1$, which implies that this data stream can be fully transmitted, when the SU accesses the system.

2) The SU has $N$ data streams, and each one has the same average arrival rate of $\lambda$. Substituting these into Equation (27), we arrive at the queue scheduling scheme characterized by $p_{i}^{*}=1 / N$, which indicates that each data stream of the SU has the same probability of being transmitted, when the SU accesses the system.

3) The SU has $N$ data streams and the average arrival rates are $\left\{\lambda_{i}=i \cdot \lambda, i=1,2, \ldots N\right\}$. Substituting these into Equation (27), the queue scheduling scheme is now characterized by

$$
p_{i}^{*}=\frac{2 \mu_{S, \text { max }}-\lambda N(1+i+N)}{2 N \mu_{S, \text { max }}} .
$$

\section{Performance Analysis}

In this section, numerical results are provided not only for characterizing the influence of the physical and the MAC layer parameters on the delay and service rate of the SU but also for benchmarking the proposed queue scheduling scheme against both the conventional Proportional as well as the Round-Robin schemes.
The physical layer parameters are detailed below. The average channel power gain (ACPG) of the PU link is set to $\gamma_{P}=5 \mathrm{~dB}$, and the outage threshold is $\beta_{P}=1 \mathrm{~dB}$. The ACPG of the SU link is set to $\gamma_{S}=6 \mathrm{~dB}$, and the outage threshold is $\beta_{S}=1 \mathrm{~dB}$; The ACPG of the PI link is set to $\gamma_{P I}=3$ $\mathrm{dB}$, while the outage threshold is $\beta_{P I}=1 \mathrm{~dB}$; ACPG of the SI link is set to $\gamma_{S I}=3 \mathrm{~dB}$, and the outage threshold is $\beta_{S I}=1$ $\mathrm{dB}$. These parameters are reasonable, since the PU/SU is most likely to be located far from the SD/PD, which results in the lower ACPG of the PI/SI link, and similarly, the PU/SU is located in the vicinity of the $\mathrm{PD} / \mathrm{SD}$, which results in a higher ACPG for the PU/SU link.

Having considered the physical layer, the parameters of the MAC layer are set up as below. Assuming that the SU has five different queuing buffers, we can set the average packet arrival rate of each queue for example to 0.01 packets/TS, 0.02 packets/TS, 0.03 packets/TS, 0.04 packets/TS, and 0.05 packets/TS, respectively. The parameters in the MAC layer of the PU, such as the average packet arrival rate $\lambda_{P}$ and the delay tolerance $T_{P}$ will be varied in order to investigate their influence on the SU's achievable performance. We vary the average packet arrival rate $\lambda_{P}$ from 0.36 packets/TS to 0.41 packets/TS in order to investigate its influence on the maximum average service rate and on the overall average delay for three different queue scheduling schemes at the SU. Three cases are considered, namely $T_{P}=4 \mathrm{TS}, T_{P}=10 \mathrm{TS}$, and $T_{P}=1000 \mathrm{TS}$

Two other queue scheduling schemes are used as benchmarkers, namely the Round-Robin scheme, where every queue of the SU has the same probability of transmitting packets stored in the buffer, and the Proportional scheme, where the transmission probabilities are arranged to be proportional to the average arrival rates at the buffers.

It may be readily seen from Fig. 2 (a) that the maximum service rate $\mu_{S \text {, max }}$ of the SU decays linearly upon increasing the average packet arrival rate $\lambda_{P}$. Furthermore, having a higher delay tolerance $T_{P}$ for the PU results in a reduced $\mu_{S \text {,max }}$. This trend is plausible, since a higher $\lambda_{P}$ results in an increased traffic load for the PU, which in turn results in the PU's prolonged occupation of the spectral band. This reduces the SU's chances of transmitting its own packets, which is directly reflected by the reduction of $\mu_{S, \max }$. Furthermore, having a reduced $T_{P}$ also implies more stringent QoS requirements, which can only be satisfied, if the PU occupies as much of the resources as possible to transmit its own data, which in turn reduces $\mu_{S, \max }$.

Observe furthermore in Fig. 2 (b) that the overall average delay of the SU is increased remarkably upon increasing $\lambda_{P}$, while a reduced $T_{P}$ brings about an increased overall delay. These trends can be interpreted using similar explanations to those of the $\mu_{S, \max }$ trends. In this graph we compared three different scheduling schemes. It can be clearly seen that our optimal scheme has a better performance than the conventional ones. Specifically, the higher the PU's traffic load - as represented by a higher $\lambda_{P}$ and lower $T_{P}$ - the higher the advantage of our optimal scheme becomes. In the case of 


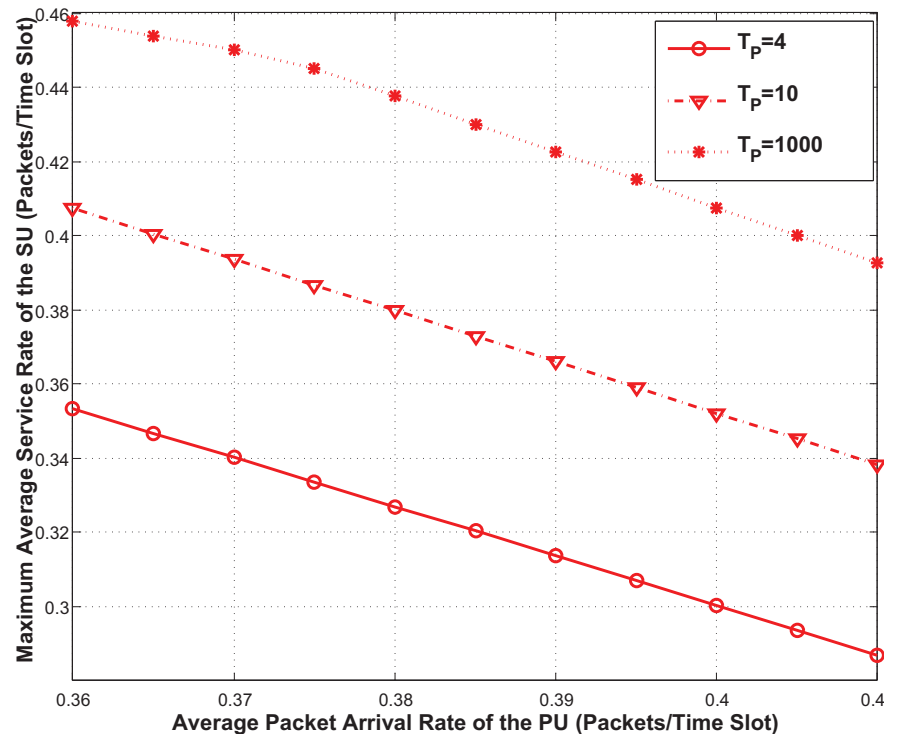

(a) Maximum average service rate of the $\mathrm{SU}$

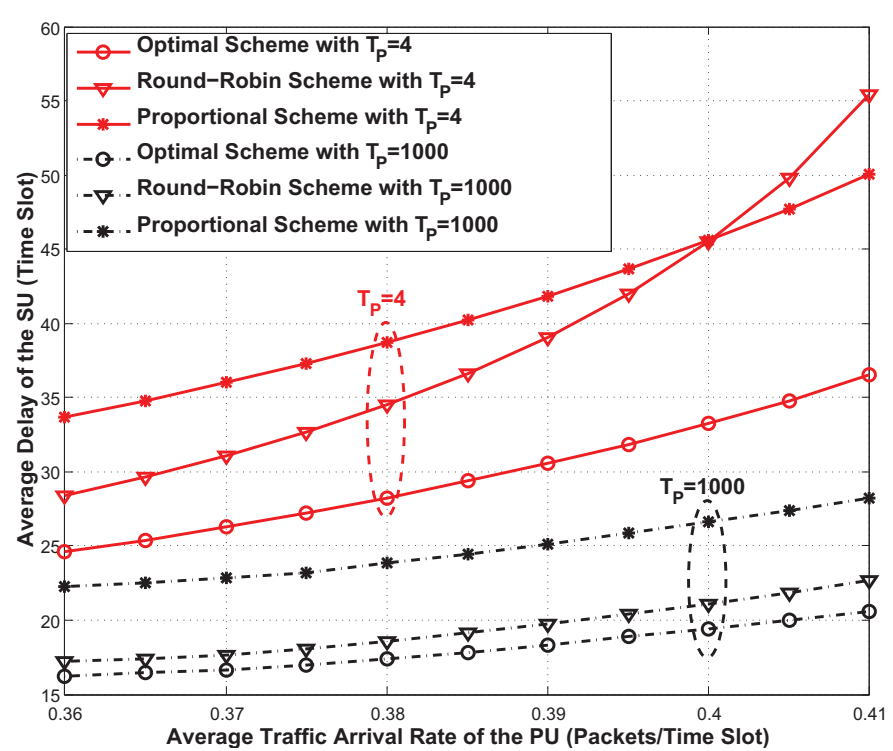

(b) Minimum overall average delay of the SU's multiple queues

Fig. 2: Service rate (a) and delay (b) of the SU versus the average packet arrival rate $\lambda_{P}$ of the PU parameterized by the delay tolerance $T_{P}$

$\lambda_{P}=0.41$ packets $/ \mathrm{TS}$ and $T_{P}=4 \mathrm{TS}$, our scheme has a $27 \%$ and $34 \%$ lower delay than the Proportional and the RoundRobin schemes, respectively.

\section{Conclusions}

A novel hybrid CR system was investigated, supporting a pair of PU source and destination as well as a pair of SU source and destination. Our hybrid scheme amalgamated the interweave and underlay paradigms for enhancing the chances of the SU to access the system. The hybrid parameter $\varepsilon$ was introduced for optimally blending the interweave and underlay paradigms, which was defined as the SU's probability of accessing the system, when the PU is still transmitting. Based on queuing theory, two problems have been solved without violating the delay constraints of the PU: (1) The optimal hybrid parameter of $\varepsilon^{*}$ was found for maximizing the average service rate of the SU; (2) The most suitable queue scheduling scheme $\left\{p_{i}^{*}, i=1,2, \ldots N\right\}$ was found for minimizing the overall average delay of the SU's multiple queues. Our numerical results characterized the influence of the physical and MAC layer parameters both on the delay and on the achievable average service rate of the SU. Finally, we benchmarked the proposed scheme against two conventional scheduling schemes. It was observed that the SU's overall average delay relying on our optimal scheme is $27 \%$ and $34 \%$ lower than that of the Proportional as well as that of the Round-Robin schemes.

\section{REFERENCES}

[1] S. Haykin, "Cognitive radio: Brain-empowered wireless communication," IEEE Journal on selected areas in communications, vol. 23, pp. 201-220, Feb, 2005.
[2] A. Goldsmith, S. A. Jafar, I. Maric, and S. Srinivasa, "Breaking Spectrum Gridlock with Cognitive Radios: An Information Theoretic Perspective", Proceedings of the IEEE, vol. 97, no. 5, pp. 894-914, May, 2009

[3] Q. Du, and X. Zhang, "Statitical QoS provisionings for wireless unicast/multicast of multi-layer video streams," IEEE Jounal on selected areas in communications, vol. 28, no. 3, April, 2010

[4] O. Simeone, Y. Bar-Ness, and U. Spagnolini, "Stable throughput of cognitive radios with and without relaying capability," in IEEE Transaction on communications, vol. 55, no. 12, December, 2007.

[5] X. Bao, P. Martins, T. Song and L. Shen, "Stable throughput analysis of multi-user cognitive cooperative systems," in Proceedings of IEEE GLOBECOM 2010, Miami, USA.

[6] A.A. El-Sherif, A.K. Sadek, and K.J.R. Liu, "Opportunistic multiple access for cognitive radio networks", IEEE Journal on selected areas in communications, vol. 29, no. 4, pp. 704-714, April, 2011

[7] L. Hanzo, P.J. Cherriman, J.Streit, Video compression and communications: from basics to H.261, H.263, H.264, MPEG4 for DVB and HSDPAstyle adaptive turbo-transceivers, John Wiley \& Sons, 2007

[8] P.V. Mieghem. Performance analysis of communications networks and systems, Cambridge University Press, 2006

[9] V. Naware, G. Mergen, L. Tong, "Stability and delay of finite-user slotted ALOHA with multipacket reception," IEEE Transactions on information theory, vol. 51, no. 7, July, 2005

[10] S. Komoella, H.D. Sherali, A. Ephremides, "Optimal scheduling in interference limited fading wireless networks," in Proceedings of IEEE GLOBECOM 2009, Hawaii, USA.

[11] R. Rao, and A. Ephremides, "On the stability of interacting queues in a multiple-access system," IEEE Transactions on Information Theory, vol.34, pp.918-930, September, 1988

[12] R. Loynes, "The stability of a queue with non-independent interarrival and service times," in Proceedings of the Cambridge philosophical society, vol.58, pp. 497-520, 1962

[13] S. Boyd, and L. Vandenberghe, Convex Optimization, Cambridge University Press, 2004 